\title{
Adaptive narrowband interference mitigation by designing UWB waveforms based on radial basis function neural networks
}

\author{
Xuebin Sun*, Bin Li, Chenglin Zhao and Yuhang Jia
}

\begin{abstract}
In order to mitigate the mutual interference between ultra-wideband (UWB) impulse radio and other existing wireless systems, a novel adaptive interference-avoiding UWB pulse, in the context of the appealing cognitive radio, is presented based on the radial basis function neural networks. Theoretical and implementation architecture for UWB pulse generator is addressed. Transmission performance in the presence of narrowband interference including tone interference is comprehensively analyzed. The presented generator can adaptively change the spectrum shape of UWB signals according to the authorized users' working states, and hence the mutual interference between UWB and other systems sharing the frequency bands can efficiently be avoided. The critical performance indication parameters, such as the SNR, SIR, and BER of UWB communication links, are also derived. Performance evaluations and comparisons between the proposed scheme and other pulse optimization methods have been provided based on the extensive numerical simulations. It is demonstrated that the presented pulse can indeed achieve much superior performance in terms of either single-link BER or anti-jamming capability.
\end{abstract}

Keywords: Ultra-wideband, Waveform design, Narrowband interference, Adaptive interference suppression, Radial basis function neural networks

\section{Introduction}

Ultra-wideband (UWB) impulse radio (UWB-IR) is a promising technology in short-range and high data rate communications, such as the wireless personal area networks. Occupying an enormous emission bandwidth, nevertheless, UWB transmitter should carefully take narrowband interference into consideration in the presence of other wireless communication systems, also for the elegant coexistence with each other. Although the U.S. Federal Communications Commission (FCC) has strictly regulated the maximum limitation of transmission power of UWB signals [1], recent studies also reveal that even UWB emission signal taking the regulated spectral may still produce unfavorable interference to other narrowband wireless communication systems [2], and simultaneously, its own transmission performance may greatly be disrupted by corresponding narrowband interference generated from these wireless services. Since traditional wireless

\footnotetext{
* Correspondence: sunxuebin@bupt.edu.cn

Key Lab of Universal Wireless Communications, MOE, Beijing University of Posts and Telecommunications, Beijing, People's Republic of China
}

communication systems always have a communication priority which means they may not change their working time or spectral characteristics to cooperate UWB devices, how to suppress interference from and to narrowband wireless communication systems is of great importance for UWB systems.

For UWB-IR system, there are mainly three kinds of resolutions to suppress narrowband interference, including a minimum mean square error (MMSE) criterion-based RAKE receiver, a notch filter, and a special pulse designing [3]. However, the RAKE receiver designing can only benefit for UWB devices to reduce the external interference from narrowband systems, while its interference to the narrowband systems cannot be mitigated at all. For a notch filter designing, the hardware implementation is rather complex. More importantly, these schemes may not change their spectral structure according to various dynamically changed spectral environments, and thus can hardly keep coexistence with other narrowband wireless services. The most effective way to mitigate mutual

\section{Springer}

(c) 2013 Sun et al.; licensee Springer. This is an Open Access article distributed under the terms of the Creative Commons Attribution License (http://creativecommons.org/licenses/by/2.0), which permits unrestricted use, distribution, and reproduction in any medium, provided the original work is properly cited. 
interference is to carefully design an adaptively interference-avoiding UWB pulse [4-7].

In [4], based on the emerging concept of cognitive radios (CRs), multiple pulse waveforms adaption is considered for generating the expected spectral notches while matching FCC spectral mask. A novel UWB pulse designing algorithm based on linear combination of different derivative functions of Gaussian pulses is proposed in [5], and the results suggested that it can achieve better BER performance and anti-jamming capability than the traditional Scholtz's monocycle. Another adaptive UWB pulse shaping method based on the orthogonal wavelet is present in [6], the CRbased technique is also introduced. The designed pulse can indeed get a relatively high spectral efficiency compared with the other methods. However, most of the waveform designing schemes presented in [4-7] just take tone interference into consideration, which makes their spectrum utilization and single-link BER performances significantly decrease as the increase of the total tone interference number. In [8], the interference with a broad frequency bandwidth can be suppressed through the pulse notch technique, however, the attenuation of designed UWB PSD in the interesting band corresponding interferences is only $50 \mathrm{~dB}$ or less, which may be still not applicable for CR scenarios considering that it cannot change its pulse spectrum to accommodate to authorized users' working state.

Motivated by the considerations above, in this article based on the novel CR concept, an adaptive interferenceavoiding UWB pulse is presented by resorting to radial basis function neural networks (RBFNN). Theoretical RBFNN architecture for UWB pulse generator is comprehensively addressed. Transmission performance in the presence of narrowband interference including tone interference is also analyzed. Key performance indications, such as the signal-to-thermal noise ratio (SNR), signal-to -interference ratio (SIR), and BER of UWB communication links, are also derived. Numerical derivations and performance evaluation have demonstrated that this UWB pulse can indeed effectively avoid interference from other wireless systems, and can adaptively change its emission spectrum shape to completely mitigate the potential interference to the authorized users.

The remainder of this article is organized as follows. Section 2 describes the adaptive interference-avoiding UWB pulse design algorithm. Theoretical performance of UWB links with narrowband interference is analyzed in Section 3. Performance evaluation and comparison between the proposed pulses and the existing method are presented. Numerical simulations are then given in Section 4. At last, we conclude the article in Section 5.

\section{RBFNN-based pulse designing}

In this article, our proposed UWB waveform designing scheme mainly abstracts pulse designing problem under different spectral constraint to be a classic interpolation in mathematics, and then we may properly adopt the RBFNN to get a novel pulse generator [9]. Due to the excellent function interpolation capability of RBFNN, our UWB pulse can have a perfect spectrum utilization efficiency over other designed pulses in [3-7], and less hardware implementation complexity in [10]. More importantly, with no change of hardware structure, the implementation algorithm can adaptively adjust the frequency spectrum to dynamically avoid authorized frequency working bands in a short time [11-13].

Figure 1 shows the network theoretical model for UWB pulse generation [9], which according to the external environment such as authorized users' working states, determine the expected optimal spectrum UWB signal $\mathbf{t}$, then set $\mathbf{t}$ as the target signal, adaptively update the parameters of the networks including network weight $\mathbf{w}$, position and waveform of base function to get the minimum error between the network output a and target signal $\mathbf{t}$ [14]. The detailed description of all components of the network model is presented in the following sections.

\subsection{Transform function $T_{i}$}

The transform function $T_{i}$ is used to generate network input $p_{i}(n)\left(i=0,1, \ldots, n_{2}\right)$, network input $p_{i}(n)$ is the basis function. Since $\mathbf{p}_{i}$ can be expressed as $p_{i}(n)(n=0,1, \ldots, N)$, then we can construct an $N \times 1$ dimensional vector $\mathbf{P}$

$$
\mathbf{P}=\left[\mathbf{p}_{0}, \mathbf{p}_{1}, \mathbf{p}_{2}, \ldots, \mathbf{p}_{n_{2}}\right]
$$

For simple analysis, we chose the Gaussian pulse as the basis function

$$
\left\{\begin{array}{c}
p_{0}(k)=1, \quad k=0,1, \ldots, N-1 \\
p_{i}(k)=\frac{1}{\sqrt{2 \pi} s_{i}} \exp \left(-\frac{f_{s}^{2}}{2 s_{i}}\left(k-\mu_{i}\right)^{2}\right), \\
i \neq 0, \quad k=0,1, \ldots, N-1
\end{array}\right.
$$

where $\mu_{i}$ and $s_{i}$ are adjustable parameters for transform function $T_{i} ; f_{s}$ is the sample frequency. We can

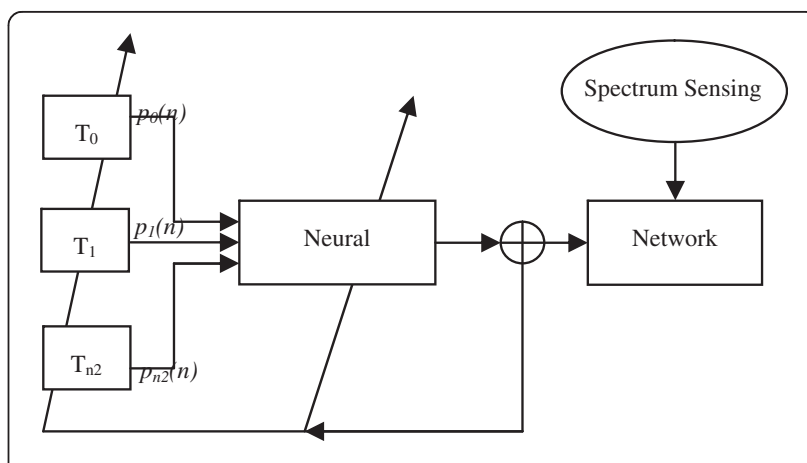

Figure 1 Network theoretical model for UWB pulse generation. 
adaptively adjust $\mu_{i}$ and $s_{i}$ to change the position and waveform of the respective basis function $p_{i}(n)$ to approach the target function in an optimal way.

\subsection{Target output vector $t$}

The target output vector $\mathbf{t}$ is the optimal frequency spectrum at the current moment. Take the template made by FCC as example, assuming that there are I kinds of authorized users, our target output vector can be expressed as $\mathbf{t}=\left\{t_{k}, k=0,1, \ldots, N-1\right\}$ and

$$
t_{k}=\sqrt{M_{\mathrm{FCC}}(f)} \times\left.\prod_{i=1}^{I}\left(1-G_{i}(f)\right)\right|_{f=k f_{s}},
$$

where $M_{\mathrm{FCC}}(f)$ is the frequency spectrum template made by FCC, $G_{i}(f)$ is a gate function and denotes that the $i$ th authorized frequency band $\left[f_{i 1}, f_{i 2}\right]$ is recognized. And $G_{i}(f)$ can be given by

$$
G_{i}(f)=\left\{\begin{array}{cc}
1 & f \in\left[f_{i 1}, f_{i 2}\right] \\
0 & \text { others }
\end{array}\right.
$$

\subsection{Network parameters updating}

To minimize the MMSE between the network output vector $\mathbf{a}$ and the target output vector $\mathbf{t}$, it is needed to adjust the network weight vector $\mathbf{w}$, and parameters $\mu_{i}$ and $s_{i}$ for transform function. We can define the mean square error as

$$
E=\frac{1}{2}\|\mathbf{a}-\mathbf{t}\|_{2}^{2}=\frac{1}{2} \sum_{i=0}^{N-1} \sum_{i=0}^{n_{2}}\left[w(i) p_{i}(k)-t(k)\right]^{2} .
$$

During the adjustment of RBFNN, the updating of the network weight vector $\mathbf{w}$ is by the Windrow-Hoff rule and parameters $\mu_{i}$ and $s_{i}$ by the steepest descent method, respectively [15].

\subsection{Output pulse}

When the network weight vector $\mathbf{w}$, parameters $\mu_{i}$ and $s_{i}$ are converged to an optimal value, the mean square error between the practical networks output $\mathbf{a}$ and the target output vector $\mathbf{t}$ come to the minimum, thus the UWB pulse in frequency domain can approach the FCC frequency spectrum template $M_{\mathrm{FCC}}(f)$ the most. We can get the output pulse in time domain.

$$
\begin{aligned}
\mathrm{s}(\mathrm{n}) & =\operatorname{IDFT}(\mathbf{a} \otimes \Theta) \\
& =\operatorname{IDFT}\left(\operatorname{purelin}\left(\mathbf{w}^{\mathrm{T}} \mathbf{P} \otimes \Theta\right)\right),
\end{aligned}
$$

where $\Theta$ is the phase response vector for users, and linear phase is chosen for our proposed pulse design, $\otimes$ denotes the piecewise product of two vectors and purelin denotes the transfer function of the neural networks, $\mathbf{z}$ is the double-side frequency spectrum conjugate and symmetry of $\mathbf{z}$, and $\mathbf{z}$ is the single-side frequency spectrum.

\section{Performance analysis with interference}

In this section, we may analysis the transmission performance of UWB signals both in absence of narrowband interference and in the presence of inevitable narrowband interference.

\subsection{Basic assumptions}

1) The BER performance is calculated for a UWB receiver over a single AWGN channel, the effect of multipath and multi-user interference is out the consideration of this research.

2) For the UWB communication architecture, the bipolar pulse amplitude modulation (BPAM) scheme is adopted in transmitter, and the optimal correlation receiver in adopted in receiver which is also is depicted in Figure 2 [11]. Besides, we also assume the perfect synchronization between the transmitter and receiver has been achieved.

3) Each of narrowband interference may have a relatively plat PSD in their working bandwidth, which is reasonable for most wireless services that employ the appealing CDMA or OFDM transmission techniques. Hence, we can conveniently calculate the power of each interference simply by $P_{\mathrm{NB}}^{i}=A_{i} B_{\mathrm{NB}}^{i}$, where $P_{\mathrm{NB}}^{i}$, $A_{i}$, and $B_{\mathrm{NB}}^{i}$, respectively, denote the power, the PSD amplitude, and the frequency working bandwidth of the $i$ th narrowband interference.

\subsection{Analysis with narrowband interference}

Since BPAM modulation is adopted in UWB transmitter, then the emission signal can be given by

$$
s_{t r}(t)=\sum_{j=-\infty}^{+\infty} d_{j} \sqrt{E_{\mathrm{TX}}} p_{0}\left(t-j T_{s}\right)
$$

where $\sqrt{E_{T X}}$ is transmitted power per bit, $p_{0}\left(t-j T_{s}\right)$ is the proposed baseband pulse, $T_{s}$ is pulse repetition time or frame time, $d_{j} \in\{+1,-1\}$ is the transmitted information data bits. After propagated in AWGN channel, the received signals can be expressed into

$$
r(t)=s_{r e}(t)+r_{\text {int }}(t)+n(t)
$$

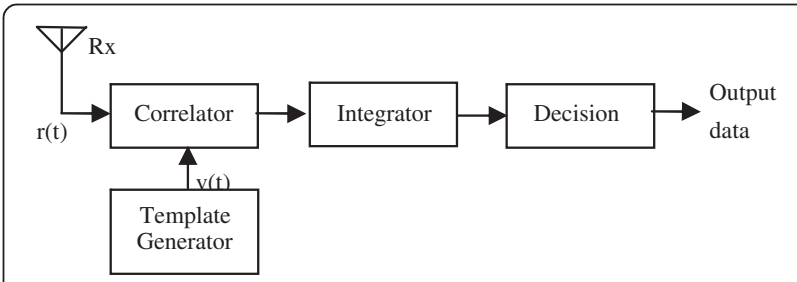

Figure 2 Block diagram of the UWB receiver. 


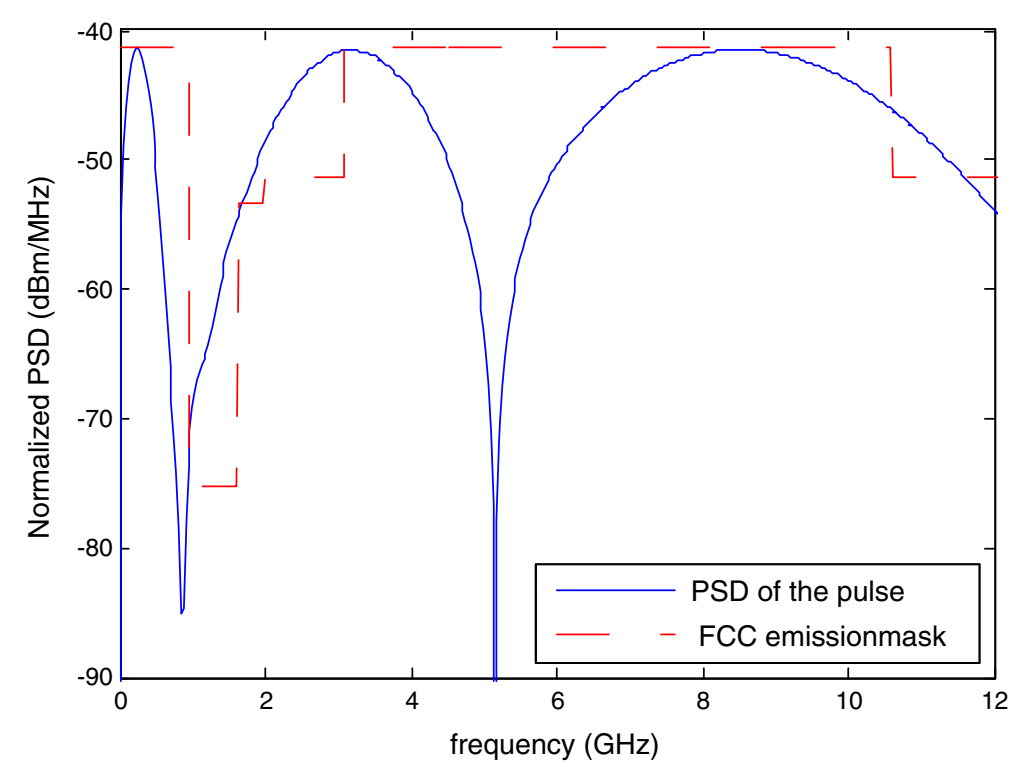

Figure 3 The spectral characteristic of the pulse designed in [5].

where

$$
s_{r e}(t)=\sum_{j=-\infty}^{+\infty} \beta d_{j} \sqrt{E_{\mathrm{Tx}}} p_{0}\left(t-j T_{s}-\tau\right)
$$

is the useful signal, while $r_{\text {int }}(t)$ and $n(t)$ represent the narrowband interference and the thermal noise, respectively. $\tau$ and $\beta$ are the propagation delay and the amplitude gain of wireless AWGN channel, respectively. The Fourier transforms of $s_{\text {re }}(t), r_{\text {int }}(t)$, and $n(t)$ can be denoted by $S_{\text {re }}(f), R_{\text {int }}(f)$, and $N(f)$, respectively. Usually, within the soft decision framework, the received signals are correlated and demodulated per frame, thus assuming in a symbol cycle $\left[0, T_{s}\right]$, the data bit $d_{j}=+1$, and we can define, during the local correlator, the Fourier transforms of the template signal as

$$
V(f)=P_{0}^{*}(f) e^{j 2 \pi f T_{S}} e^{j 2 \pi f \tau}
$$

then the sample output of the correlator at the moment $T_{s}$ can be given by

$$
Z=\int_{f_{L}}^{f_{H}} R(f) V(f) d f=Z_{\text {use }}+Z_{\text {int }}+Z_{\text {ther }}
$$

where $Z, Z_{\text {use }}, Z_{\text {int }}$, and $Z_{\text {ther }}$ respectively, represent the decision variable, the useful signal component, the interference component, and the thermal noise component. Furthermore, the energy of useful signal component can be calculated by

$$
\begin{aligned}
E_{\mathrm{use}}= & \left(Z_{\mathrm{use}}\right)^{2} \\
= & E\left\{\beta \sqrt{E_{\mathrm{TX}}} \int_{f_{L}}^{f_{H}}\left[P_{0}(f) e^{-j 2 \pi f T_{s}} e^{-j 2 \pi f \tau}\right]\right. \\
& \left.\quad\left[P_{0}^{*}(f) e^{j 2 \pi f T_{s}} e^{j 2 \pi f \tau}\right] d f\right\}^{2} \\
= & \beta^{2} E_{\mathrm{TX}}\left(\int_{f_{L}}^{f_{H}} P_{0}(f) P_{0}^{*}(f) d f\right)^{2} \\
= & \beta^{2} E_{\mathrm{TX}} E_{p}^{2}
\end{aligned}
$$

where $E_{p}$ is the in-band power of the proposed UWB pulse. Also, the received interference energy can be expressed into

$$
\begin{aligned}
E_{\text {int }} & =Z_{\text {int }}^{2} \\
& =E\left\{\int_{f_{L}}^{f_{H}} R_{\text {int }}(f) P_{0}^{*}(f) e^{j 2 \pi f T_{s}} e^{j 2 \pi f \tau} d f\right\}^{2} \\
& =E\left\{\int_{f_{L}}^{f_{H}} R_{\text {int }}(f) P_{0}^{*}(f) d f \int_{f_{L}}^{f_{H}} R_{\text {int }}^{*}(f) P_{0}(f) d f\right\}
\end{aligned}
$$

Since we made an assumption that each narrowband system has a plat PSD, we mainly consider two types of interference:

\section{Case A: tone signal interference}

In Case $A, P_{O}(f)$ and $P_{O} *(f)$ in (13) are constants, thus further mathematic treatment of (13) may lead to

$$
E_{\mathrm{int}}=\sum_{i} P_{\mathrm{NB}}^{i}\left|P_{0}^{2}\left(f_{\mathrm{NB}}^{i}\right)\right|
$$

where $\left|P_{0}^{2}\left(f_{\mathrm{NB}}^{i}\right)\right|$ is the PSD value of the proposed UWB pulse at the working frequency of the $i$ th tone interference. 


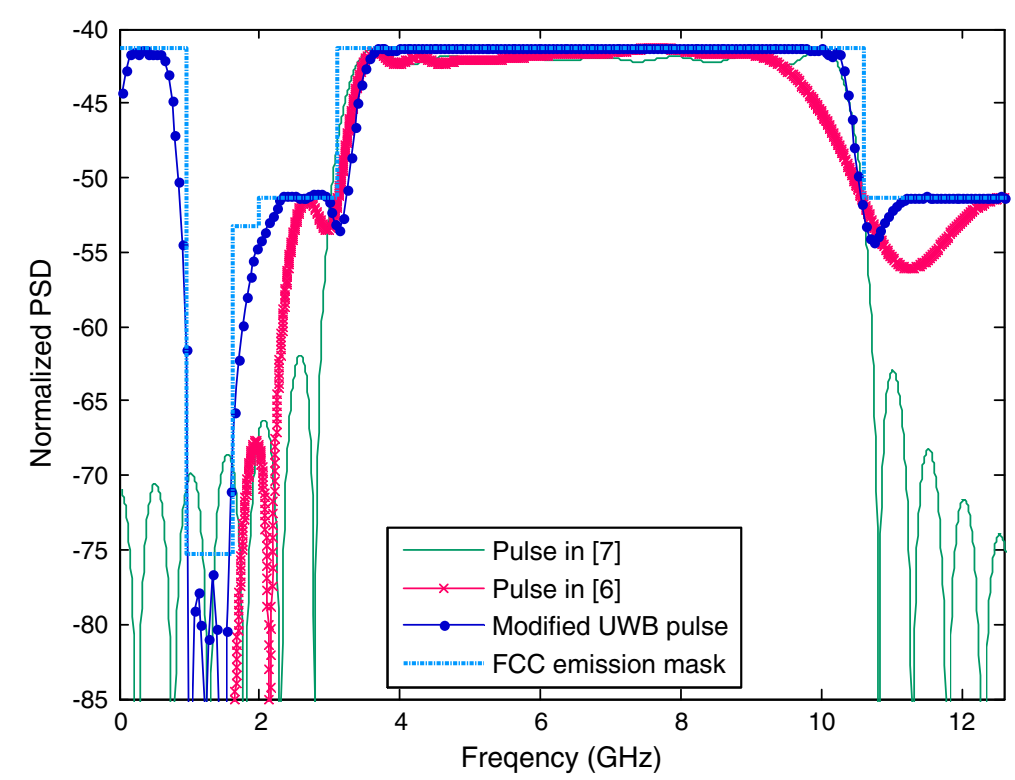

Figure 4 The spectral characteristic of designed UWB pulses under FCC spectral emission mask.

\section{Case B: narrowband signal interference with working} bandwidth $B_{N B}^{i}$

In this case, each interference signal is a narrowband signal with working frequency bandwidth $B_{\mathrm{NB}}^{i}$. Given that each interference has a plat PSD, (13) can be further expressed into

$$
E_{\mathrm{int}}=\sum_{i} P_{\mathrm{NB}}^{i} \int_{B_{\mathrm{NB}}^{i}}\left|P_{0}^{2}(f)\right| d f
$$

For the variable $Z_{\text {ther, }}$ it is apparent that its mean is equal to zero, and the corresponding variance can be given by

$$
\sigma_{\text {ther }}^{2}=E\left\{\int_{f_{L}}^{f_{H}} N(f) P_{0}^{*}(f) d f\right\}^{2}=\frac{N_{0}}{2} E_{p}
$$

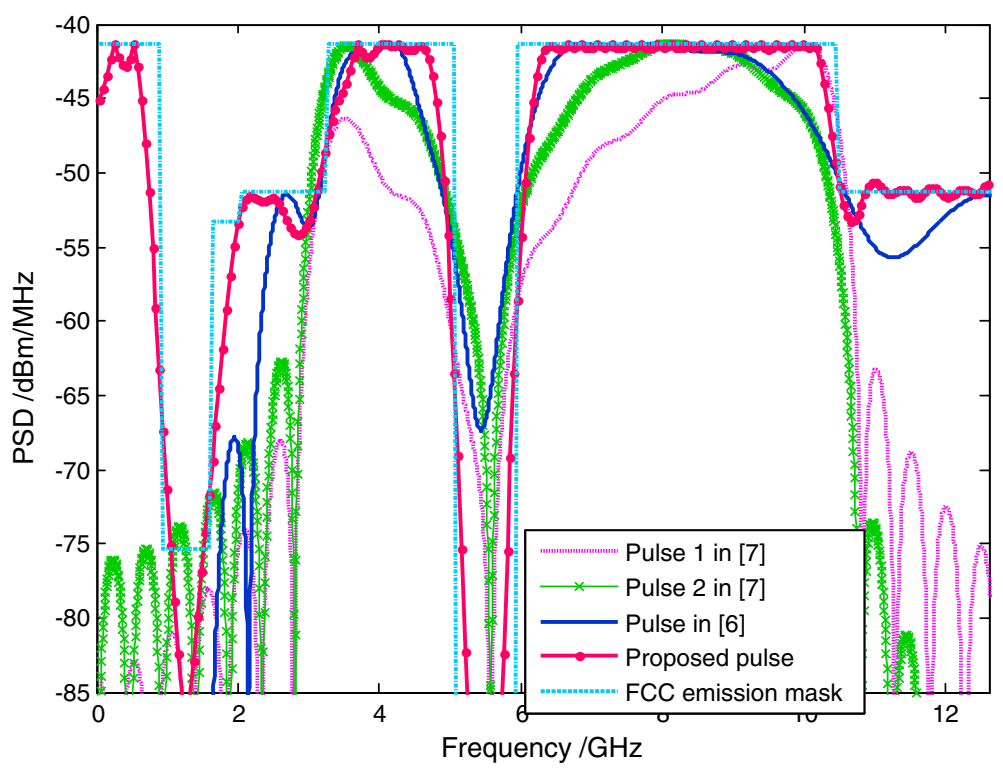

Figure 5 The spectral characteristic of designed UWB pulses with spectral notch. 


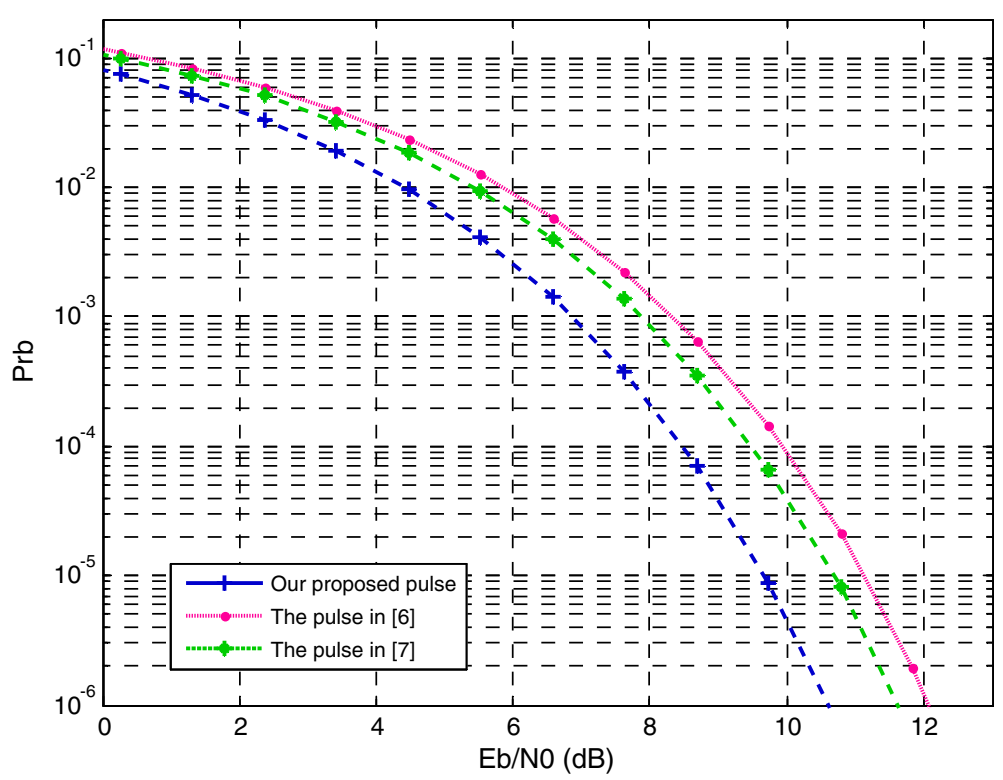

Figure 6 The bit error probability of the UWB pulses with tone interference. (a) $E b / N O=10 \mathrm{~dB}$; (b) Eb/NO $=20 \mathrm{~dB}$.

Besides, SIR and SNR can be given by

$$
\mathrm{SIR} \triangleq \frac{E_{\mathrm{use}}}{E_{\mathrm{int}}}=\left\{\begin{array}{ll}
\frac{\beta^{2} E_{\mathrm{TX}} E_{p}^{2}}{\sum_{i} P_{\mathrm{NB}}^{i}\left|P_{0}^{2}\left(f_{\mathrm{NB}}^{i}\right)\right|} & \text { Case } A \\
\frac{\beta^{2} E_{\mathrm{TX}} E_{p}^{2}}{\sum_{i} P_{\mathrm{NB}}^{i} \int_{\mathrm{B}_{\mathrm{NB}}^{i}}\left|P_{0}^{2}(f)\right| d f} & \text { Case } B
\end{array} .\right.
$$

$$
\mathrm{SNR}=\frac{E_{\mathrm{use}}}{\sigma_{\text {ther }}^{2}}=\frac{2 \beta^{2} E_{\mathrm{TX}} E_{p}}{N_{0}}
$$

\subsubsection{Comparison with the pulse designed by Zhou et al.}

In [5], a UWB pulse based on CR with in-band interference suppression is designed by Zhou et al., whose spectral characteristic is showed in Figure 3. In fact, the corresponding PSD of generated pulse may not exactly comply with FCC emission mask, and it is noted that this spectral mismatch may produce serious interference to the other wireless services. Omitting that point, it is proved that the pulse has a better single-link BER performance in AWGN and anti-jamming capability compared to the other conventional waveforms such as Scholtz's monocycle. The comparison of BER performance analysis with narrowband interference between the pulse in [5] and our proposed pulse is presented as follows.

We define the received energy of the above reference pulse as $E_{b}=\beta^{2} E_{\mathrm{TX}} E_{p . z l l}$, and assuming that each $P_{\mathrm{NB}}^{i}$ is identical, define the total energy of the interference as $E_{i}=P_{\mathrm{NB}}^{i}$, take the Case $B$ as example, then the SIR and
SNR of UWB system which adopts the pulse in [5] can be given by

$$
\begin{aligned}
\operatorname{SIR}_{z l l} & =\frac{E_{b} E_{p . z l l}}{E_{i} \sum_{i} \int_{B_{\mathrm{NB}}^{i}}\left|P_{z l l}^{2}(f)\right| d f} \\
\operatorname{SNR}_{z l l} & =\frac{2 E_{b}}{N_{0}}
\end{aligned}
$$

And let us define SNR gain as

$$
\gamma=\frac{E_{p}}{E_{p . z l l}}
$$

Then, the SIR gain can be given by

$$
\eta=\frac{\operatorname{SIR}}{\operatorname{SIR}_{z l l}}=\gamma^{2} \sum_{i} \frac{\int_{B_{\mathrm{NB}}^{i}}\left|P_{0}^{2}(f)\right| d f}{\int_{B_{\mathrm{NB}}^{i}}\left|P_{z l l}^{2}(f)^{z l l}\right| d f}
$$

To calculate the parameter $\gamma$, we define the spectrum utilization ratio as

$$
\varphi=\frac{\int_{B_{\mathrm{UWB}}}\left|P^{2}(f)\right| d f}{\int_{B_{\mathrm{UWB}}} M_{\mathrm{FCC}}(f) d f} \times 100 \%
$$

Thus (22) can be rewritten as

$$
\gamma=\frac{\varphi_{P_{0}}}{\varphi_{P_{z l l}}}
$$

where $\varphi_{P_{0}}$ and $\varphi_{P_{z l l}}$ are the spectrum utilization ratios of our proposed pulse and the pulse in [5]. 
Then with soft decision, take Case $B$ as example, the BER of the pulse can be given by

$$
\operatorname{Pr}_{b}^{(z l l)}=Q\left(\sqrt{\left[\left(\frac{E_{b} E_{p} . z l l}{E_{i} \sum \int_{B_{N}^{i} B}\left|P_{z l l}^{2}(f)\right| d f}\right)^{-1}+\left(\frac{2 E_{b}}{N_{o}}\right)^{-1}\right]-1}\right)
$$

The BER of our proposed pulse can be given by

$$
\operatorname{Pr}_{b}^{\text {(this })}=Q\left(\sqrt{\left.\left[\left(\eta \frac{E_{b} E_{p . z l l}}{E_{i} \sum_{i} \int_{B_{\mathrm{NB}}^{i}}\left|P_{z l l}^{2}(f)\right| d f}\right)^{-1}+\left(\gamma \frac{2 E_{b}}{N_{0}}\right)^{-1}\right]-1\right)}\right.
$$
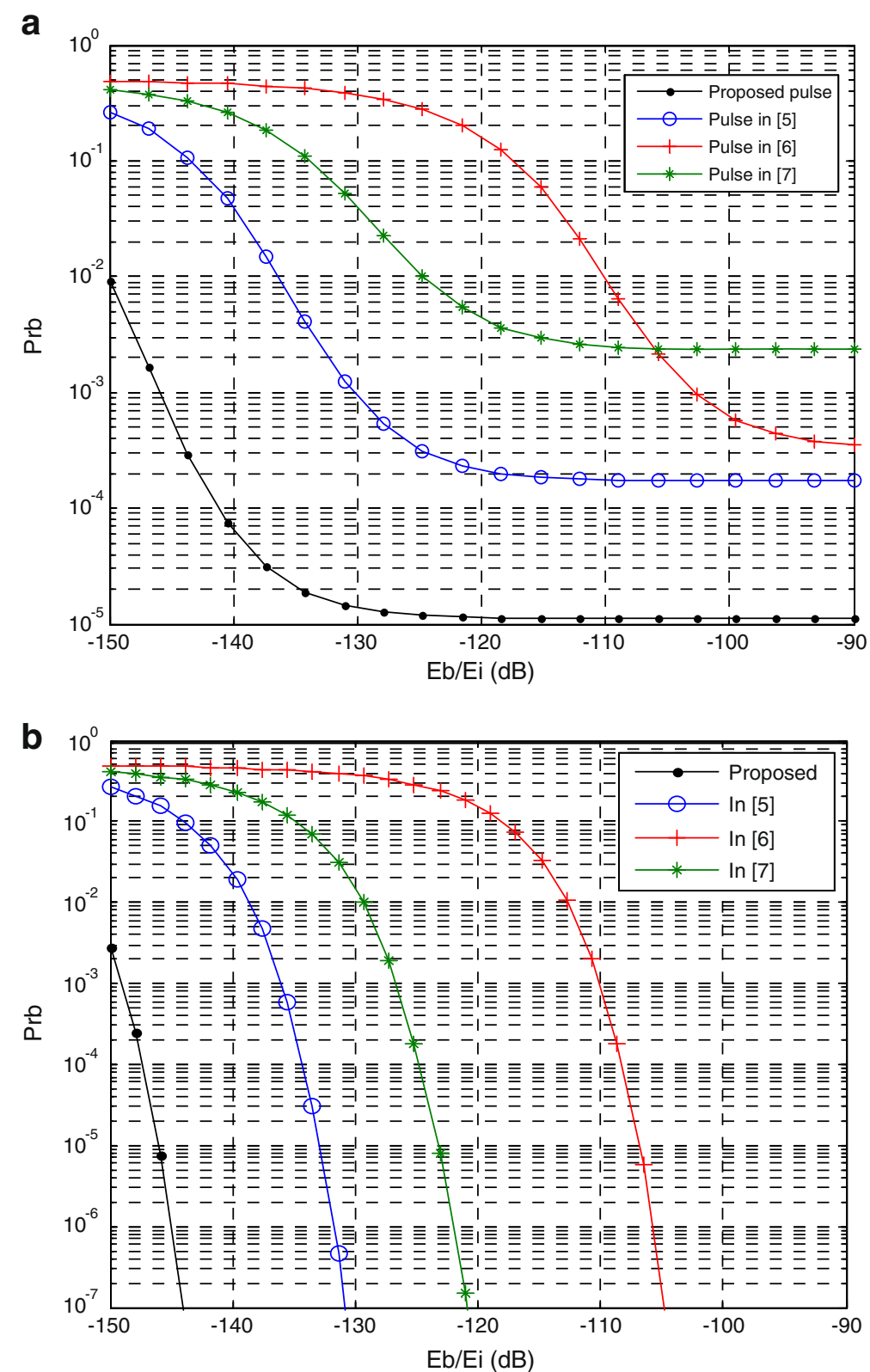

Figure 7 The error bit probability without narrowband interference. 


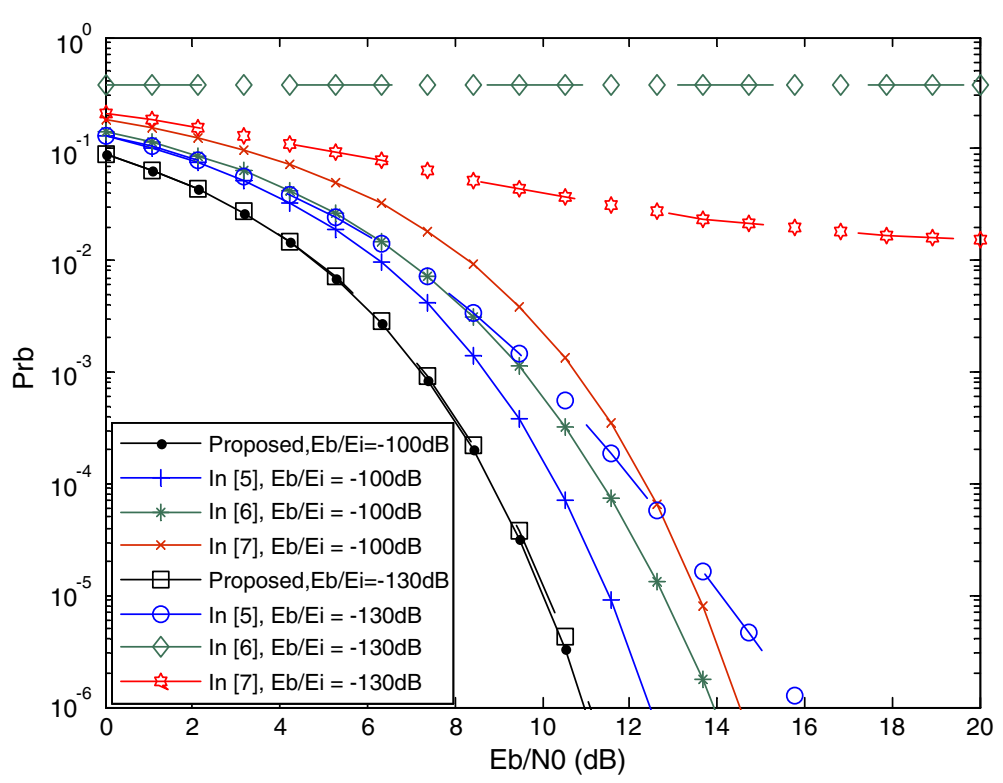

Figure 8 The bit error probability of the UWB pulses with tone interference.

\section{Simulation conditions and results}

To comprehensively evaluate the transmission performance of our pulse presented in the presence or absence of the narrowband interference, we also give the two additional UWB pulse designing techniques, respectively, based on the orthogonal wavelet in [6] and the Gate function in [7]. The designed spectral characteristic of these UWB waveforms has been illustrated in Figures 4 and 5, respectively, for two different scenes. We first note that, for the windowing-based pulse design scheme in [7], the low-frequency band from 0.99 to 1.66 $\mathrm{GHz}$ of the regulatory FCC mask may not be utilized any more, greatly restricting some significant UWB applications such as through-wall sensing. Besides, from the simulation result in Figure 4, both the generated UWB waveform cannot fully use the low frequency below $0.99 \mathrm{GHz}$. Nevertheless, it is clearly seen that our presented UWB pulse can essentially utilize the whole spectral mask.

On the other hand, when avoiding specific frequency band to mitigate mutual interference, the UWB waveforms are also plotted together in Figure 5. It is obvious that, for windowing-based pulse design scheme in [7], the spectrum utilization of the generated waveforms may considerably be reduced from Figure 5. Similar observation can be made on the orthogonal wavelet-based UWB waveforms. So, the spectral notch may greatly degrade transmission performance of UWB systems. More important, the generated spectral notch is not sufficient to mitigate mutual interference given the maximum spectral attenuation can only approach $25 \mathrm{~dB}$. In comparison, our present UWB pulse can produce the effec- tive spectral notch with a depth of $95 \mathrm{~dB}$, which can completely mitigate the inference even when multiple UWB users are taken into consideration.

\subsection{Without narrowband interference}

When the narrowband interference is absent, the receiving performance under the optimal correlator structure is only related with the SNR. Accordingly, the bit error probability can be calculated by

$$
\operatorname{Pr}_{b}=Q\left(\sqrt{\varphi \frac{2 E_{b}}{N_{0}}}\right)
$$

where the $E_{b}=\beta^{2} E_{\mathrm{TX}} E_{p . \mathrm{FCC}}$, and $E_{p \text {.FCC }}$ is the power of the FCC integrated from 0 to $10.6 \mathrm{GHz}$, thus the key factor of the UWB pulse related to the bit error probability is only the spectrum utilization ratio $\varphi$. Based on (23) and from the numerical simulations, the spectrum utilization of UWB pulse in [6,7] and our proposed are $69.8,77.5$, and $97.9 \%(N=32)$, respectively. Correspondingly, the bit error probability of three pulse in absence of narrowband interference is depicted in Figure 6, from which we can obviously note that, for the single-link, BER performance of our proposed pulse is much superior to the other pulses. From this simulation shown in Figure 7, SNR advantage of our presented UWB pulse to that of in [7] is about $2.5 \mathrm{~dB}$.

\subsection{With narrowband interference}

If we take the practical narrowband interference into consideration, the transmission performances of UWB waveforms that have adopted specific spectral avoidance 

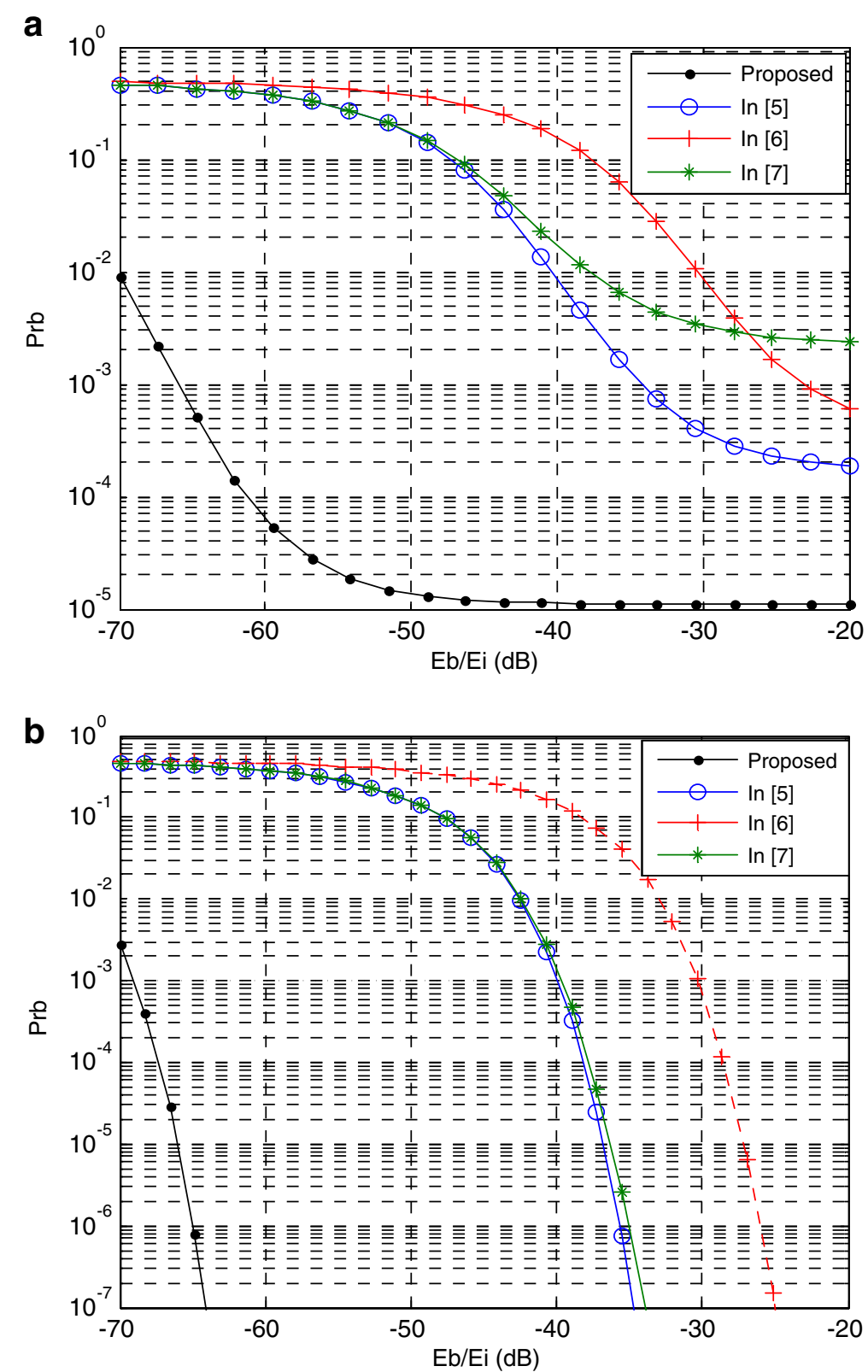

Figure 9 Bit error probability of UWB pulses with narrowband interference. (a) $E b / N O=10 \mathrm{~dB}$; (b) $E b / N O=20 \mathrm{~dB}$.

have also been given in Figures 6 and 8. Without loss of generality, for each UWB pulse, an avoiding spectral band centralized at $5.8 \mathrm{GHz}$ is adopted. For our proposed method, taking the equivalent order $N=32$ in our experiments. Based on numerical simulations, the spectrum utilization when taking the narrowband interference may slightly be reduced to $90 \%$ under the FCC spectral emission mask. Meanwhile, we also noted that spectrum utilization of other existing pulses may also be decreased much seriously, such as in [6,7], the spectrum utilization ratios are reduced to 58 and $40 \%$, respectively. On the other hand, although spectrum utilization of the
UWB pulse introduced in [5] is about 64\%, this emission waveform can hardly be applied given the obvious spectral mismatch. As analyzed in Section 3, BER performance of these UWB pulses can be derived as is shown in Figure 8. Notice that the narrowband bandwidth is chosen to be $100 \mathrm{MHz}$.

For the tone signal interference in Case $A$, as is mostly considered when evaluate the performance of the optimal shaped pulses, the narrowband interference to UWB system is relatively limited, which can obviously be seen from Figure 6. The simulated $E b / E i$ range may changes from -150 to $-90 \mathrm{~dB}$. If we set $E b / N O$ to $10 \mathrm{~dB}$, then the 


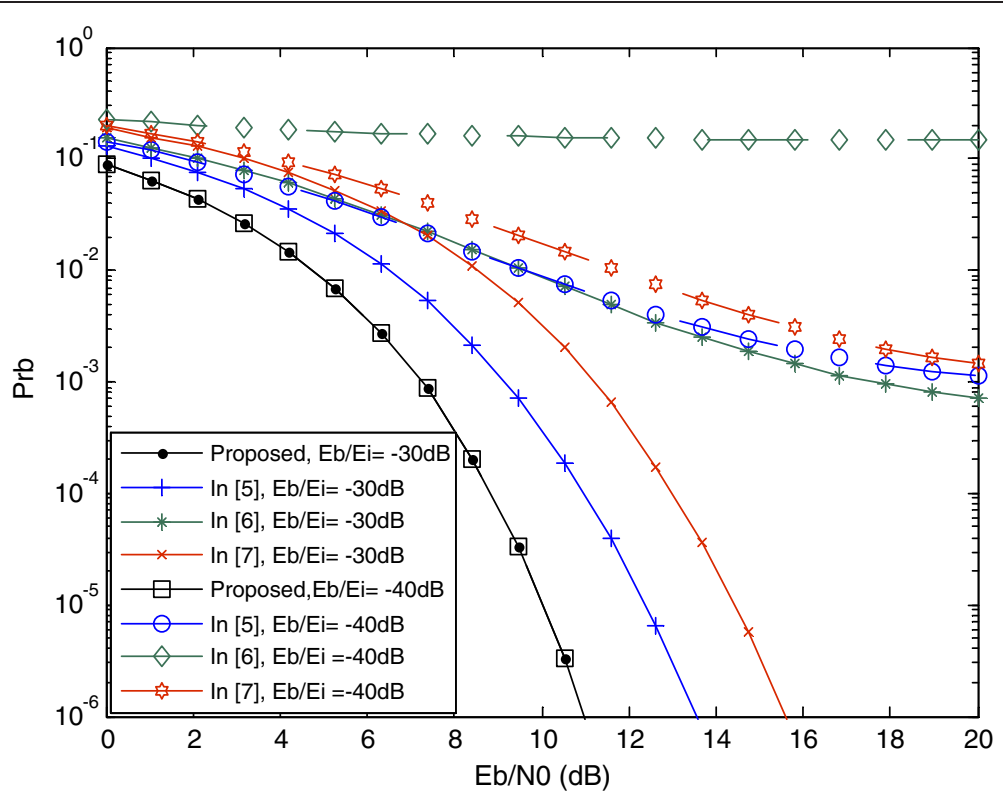

Figure 10 The bit error probability of the UWB pulses with narrowband interference.

total interference to UWB receivers can be omitted when $E b / E i$ becomes larger than $-90 \mathrm{~dB}$, no matter what kind of emission pulse is adopted. Besides, it is obvious to find that all these BER curves may converge to certain steady constants with the increasing of Eb/Ei. For example, for our propose pulse and the other pulses in [5-7], their converged BER values are $1 \times 10^{-5}, 9 \times 10^{-3}$, $7 \times 10^{-3}$, and $9 \times 10^{-2}$, respectively. Given that the achieved spectrum utilization of the four pulses are 90 , 64,58 , and $40 \%$, with the increasing the $E b / E i$, the corresponding SIR may become smaller and smaller, and hence BER performance may gradually approach to that of $E b / N O=10 \mathrm{~dB}$. Correspondingly, another important parameter in Figure 6a is the value of $E b / E i$ over which the BER curves tend to become plat or keep invariant, which can conveniently be defined as the threshold $E b / E i$ denoted by $C_{E b / E i}$. Indeed, this threshold $E b / E i$ may effectively reflect the corresponding anti-jamming capability. Specifically, the larger $C_{E b / E i}$ is, the stronger the anti-jamming capability is. From Figure 6, we also noted that $C_{E b / E i}$ of our proposed pulse and the existing pulses in [5-7] are $-130,-120,-90$, and $-115 \mathrm{~dB}$, respectively. Also, the attenuation depth in spectral avoiding band can be account for the difference of the $C_{E b / E i}$, which has been indicated by Equations (25) and (26). From Figure 7 , the spectrum attenuation depths of our proposed pulse and the existing pulses in [5-7] are -100 , $-90,-65$, and $-85 \mathrm{dBm} / \mathrm{MHz}$, respectively.

When the Eb/NO is set to $20 \mathrm{~dB}$, the narrowband interference from external tone signal may become much smaller as we can see from Figure $6 \mathrm{~b}$, in which the BER curves may not converge any more since SNR is large enough in this case, and the single-link BER performance is only related to attenuation depth in corresponding spectral avoiding band. The result is more obvious that our proposed pulse performance is the best, and the less well is the pulse in [5], the pulse in [6] may behave the worst.

In Figure 8, another relationship between the achieved $\mathrm{BER}$ and $E b / N O$ is also provided, with $E b / E i$ is fixed to -100 and $-130 \mathrm{~dB}$, respectively. When $E b / E i=-100 \mathrm{~dB}$, all of the UWB receivers may suffers no or little influence from this tone interference, and hence the corresponding BER performance is only related to the spectrum utilization ratio and $E b / N O$. When $E b / E i=$ $-130 \mathrm{~dB}$, the convergent BER of UWB pulse in [6] is a constant about 0.4 which means that the UWB system cannot work anymore; BER of the pulse presented in [7] is converged to 0.08 as the $E b / N O$ increasing, while our proposed pulse performs with just a little influence from this tone interference.

Differentiated from the tone interference, with a bandwidth of $100 \mathrm{MHz}$ and centralized at $5.8 \mathrm{GHz}$, another more general narrowband signal is also considered as the external interference to UWB receivers in this experiment. Accordingly, transmission performance of different UWB pulses with spectral avoidance has also been presented in Figures 9 and 10. The analysis is much similar to that of the tone interference situations, nevertheless, it is also noted that UWB receivers is much more immune to narrowband interference than the tone interference. Correspondingly, the BER performance can be also derived from Equations (25) and (26). In this simulation, the $E b / E i$ is varied from -70 to $-20 \mathrm{~dB}$ as is 
illustrated in Figure 9a,b. Much similar to the case of tone interference, all the four BER curves will converge to different steady value. Specifically, the converging values of our proposed pulse and the existing pulses in [5-7] are as the same as the case with tone interference, which is mainly because the interference can be still omitted when $E b / E i$ is larger than $-20 \mathrm{~dB}$. However, the threshold values $C_{E b / E i}$ are considerably changed, which respectively are $-50,-25,-20$, and $-25 \mathrm{~dB}$. When $E b / N 0$ is set to $20 \mathrm{~dB}$, BER performance is also mainly related to the attenuation depth in avoiding spectral bands. Since the generated notches of [5,7] corresponding to narrowband interference keep almost the same, their BER performances are also very close as well. It is apparent that, from Figures 9 and 10, our proposed pulse still behaves the best because of its largest attenuation spectral depth, greatly suppressing the narrowband interference and improving the UWB receiver SNR. The relationship between BER and $E b / N O$ with the fixed $E b / E i$ of -30 and $-40 \mathrm{~dB}$, respectively, is presented in Figure 10. It is also demonstrated from this numerical simulations that our proposed UWB pulse has the strongest anti-jamming capability, which can totally mitigate the narrowband interference and hence the change of $E b / E i$ may slightly affect final BER performance. In comparison, although the produced pulses in $[5,7]$ behave not bad when $E b / E i$ is $-30 \mathrm{~dB}$, both of their BER value may be always above $10^{-3}$, while our proposed pulse behaves as well as in absence of this narrowband interference.

\section{Conclusion}

As an underlying spectral accessing technique, UWB has always been confronted with stringent emission limitation from the radio management. Accordingly, the spectral coexistence between UWB and other wireless services has long been remained as one challenge research area. The CR-based UWB transmission scheme may provide an attractive solution for the mutual interference mitigation issue. As a consequence, the UWB waveform designing that can fully utilize spectral energy, and meanwhile can also effectively avoid specific band, has greatly been advanced in recent years. In this study, a novel adaptive interference-avoiding UWB waveform is suggested. The algorithm to design UWB pulses based on RBFNN and $\mathrm{CR}$ is studied. Three cases of the external interference, tone interference, narrowband interference with its working bandwidth of $100 \mathrm{MHz}$ are considered. From the numerical simulations and thorough performance evaluation, it is shown that our proposed pulse-indeed behaviors much better than the other pulse designing schemes from aspects of both the single-link BER performance and the anti-jamming capability.

\section{Acknowledgments}

This research was supported by the Fundamental Research Funds for the Central Universities (2012RC0103), the National Natural Science Foundation of China $(60902046,60972079,61271180)$, and by the National major special science and technology project of China (2011ZX03005-002, 2012ZX03001022).

Received: 18 January 2013 Accepted: 12 February 2013 Published: 2 April 2013

\section{References}

1. Federal Communications Commission (FCC), Revision of part 15 of the commission's rules regarding ultra-wide band transmission systems, First Report and Order, ET Docket 98-153, FCC 02-48, 2002. April

2. Z Li, WX Zou, B Li, Z Zhou, XJ Huang, Analysis on coexistence of ultra wideband with OFDM-Based communication systems. IEEE Trans. Electromagn. Compat. 53(3), 823-830 (2011)

3. SY Xu, QH Yang, KS Kwak, Suppression of IEEE 802.11a interference using SVD-based algorithm for DS-UWB systems in wireless multipath channels. AEU-Int. J. Electron. C 67(10), 700-704 (2007)

4. HG Zhang, XF Zhou, KY Yazdandoost, I Chlamtac, Multiple signal waveforms adaptation in cognitive ultra-wideband radio evolution. IEEE J. Sel. Areas Comm. 24(4), 878-884 (2006)

5. LL Zhou, HB Zhu, NT Zhang, Iterative solution to the notched waveform design in cognitive ultra-wideband radio system. Prog. Electromagn. Res. (PIER) 75, 271-284 (2007)

6. XL Wu, XJ Sha, C Li, NT Zhang, Orthogonal wavelet based dynamic pulse shaping for cognitive ultra-wideband communications, in Proceedings of the IEEE Global Telecommunication Conference (GLOBECOM'08), vol.10, New Orleans, LA, USA, 2008, pp. 772-786

7. WH Zhang, HB Shen, ZQ Bai, K Kyung-sup, A novel NBI suppression scheme in UWB ranging system. IEICE Trans. 90-A(11), 2439-2441 (2007)

8. HB Shen, WH Zhang, KS Kwak, Cognitive implementation of chirp waveform in UWB systems. IEICE Trans. 91-B(1), 147-150 (2008)

9. B Li, Z Zhou, WX Zou, A novel adaptive spectrum forming filter: application in cognitive ultra-wideband. Sci. China Inf. Sci. 53(12), 2584-2599 (2010)

10. K Ohno, T Ikegami, Interference mitigation study for UWB radio using template waveform processing. IEEE Trans. Microw. Theory 54, 1782-1792 (2006)

11. RY Yang, Z Zheng, An adaptive interference-avoiding system for DS-UWB cognitive radio, in Proceedings of the First International Conference on Communication Network, China (CHINACOM2006), vol.1, Beijing, China, 2006, pp. 1-5

12. A Rabbachin, TQS Quek, PC Pinto, I Oppermann, MZ Win, Non-coherent UWB communication in the presence of multiple narrowband interferers. IEEE Trans. Wirel. Commun. 9(11), 3365-3379 (2010)

13. B Maria-Gabriella Di, G Guerino, Understanding Ultra-Wideband Radio Fundamentals (Prentice Hall, Upper Saddle River, NJ, 2004)

14. B Li, Z Zhou, W Zou, Interference mitigation between ultra-wideband sensor network and other legal systems. EURASIP J. Wirel. Commun. Netw. (JWCN) 2010 (2010). Article ID 290306

15. F Guo, YQ Zhuang, A new UWB pulse design algorithm for in-band interference suppression. Acta. Photonica. Sinic. 6, 1345-1348 (2006)

doi:10.1186/1687-1499-2013-94

Cite this article as: Sun et al:: Adaptive narrowband interference mitigation by designing UWB waveforms based on radial basis function neural networks. EURASIP Journal on Wireless Communications and Networking 2013 2013:94. 